\title{
A Micromachined Permalloy Magnetic Actuator Array for Micro Robotics Assembly Systems
}

\author{
Chang Liu, Thomas Tsao, Yu-Chong Tai, Wenheng Liu ${ }^{+}$, Peter Will ${ }^{+}$, Chih-Ming Ho ${ }^{*}$ \\ Electrical Engineering, California Institute of Technology, Pasadena, California 91125 \\ ${ }^{+}$ISI, The University of Southern California, Marina del Rey, California 90292 \\ * MANE, The University of California, Los Angeles, California 90024
}

\begin{abstract}
Arrays of permalloy magnetic actuators have been studied for the use as precision micro robotics assembly systems. The actuator arrays have been tested for lifting and moving silicon and glass chips. The actuator unit consists of a permalloy plate $1 \mathrm{~mm} \times 1 \mathrm{~mm} \times 5$ $\mu \mathrm{m}$ in size together with polysilicon bending supports. Experimentally, it can lift a $87 \mu \mathrm{N}$ (or $8.88 \mathrm{mg}$ ) force under a magnetic field of approximately $2 \times 10^{4} \mathrm{~A} / \mathrm{m}$. A proposed synchronous driving mode has been observed, and both translation and rotation of a silicon chip has been demonstrated.
\end{abstract}

\section{INTRODUCTION}

In current micro fabrication facilities, millimetersized objects, such as micro electronics chips, multiple chip modules (MCM) and read/write heads for harddisk drives, are handled and assembled using macroscale machinery. The incompatibility in sizes would naturally add difficulties to micro chip manipulation. The goal of this work is to seek for a mechanism of using a two dimensional intelligent micro robotics assembly system for performing such tasks as holding, moving, orienting and sorting small objects. The envisioned micro assembly system is a surface full of unit manipulators positioned and operated in a systematic manner, to precisely and effectively maneuver micro objects that ride above its surface. Assembly using such a system would enjoy the benefits of parallel processing, among others, so that throughput will increase and assembly quality may improve [1]. At the current stage, there are two challenges for the development of such a micro system. The first is to build mathematical and computational models that explore the feasibility, efficiency and limitation of object maneuvering $[1,2,3]$. Nowadays, it is already possible to construct and simulate complex system functions using established computerized modeling tools, A second challenge, which is the focus of this paper, is the fabrication of successful unit actuators that fulfill the assembly-system requirements. For example, one challenging requirement is the load capacity. Weights of today's typical micro chips are on the order of mg (tens of $\mu \mathrm{N}$ ) and they need to be lifted vertically by ten's of $\mu$ min during assembly.

Before discussing unit-actuators, it is important to introduce two fundamental modes of array actuator transportation - synchronous and asynchronous driving [1]. In the synchronous driving mode, all actuators under the loading of one object move in unison with identical gaits. Each driving cycle consists of two phases - one in which the power to the actuator is supplied, another in which the actuator power is removed. The resultant movements of the object in these two phases can be designed to be different; therefore a net step movement can be gained through each complete cycle. The asynchronous driving mode is more complex; it requires that at least two spatially separated groups of actuators be turned on and off at different time, holding and driving the object alternately. Asynchronous driving could be a more efficient method compared with the synchronous one, but it will invariably require that actuators be individually controllable. Synchronous actuators, on the other hand, offers much simpler actuator configurations at the potential expense of speed.

Several types of unit actuators have been explored. Recently, Akiyama et. al. reported an electrostaticdriven Scratch Drive Actuator (SDA) for generating lifting forces of tens of $\mu \mathrm{N}$ and to reach upto $100 \mu \mathrm{m}$ in height[4]. Using fluid forces, Konishi and Fujita have demonstrated lifting $0.69 \mathrm{mg}$ [5], and Pister el. al. achieved lifting several mg [6]. Using thermal-bimorph cantilever actuators, Ataka et. al. [7]constructed a biomimetic micro motion system to transport a load of $2.4 \mathrm{mg}$.

On the other hand, magnetic actuation is known to generate strong and long-range forces, and different types of magnetic interactions have been explored for micro actuation [8 -12]. Kim el. al. has demonstrated the levitation and transportation of a small high-Tc superconductor object [13]. Here, we would like to show that a permalloy actuator system is also promising. Judy et. al. demonstrated a permalloy/polysilicon flexure structure for large in-plane movement [14]. A micromachined permalloy magnetic flap, with features such as large out-of-plane movement and strong force, has been realized at Caltech for controlling delta-wing airfoils[15]. In the current study, we adopted the previously developed actuation scheme to make 'unit actuators with a target of demonstrating the synchronous driving mode only.

\section{DESIGN AND FABRICATION}

The structures of an actuator, a so called magnetic flap, is shown in Fig. 1. It consists of a suspended polysilicon plate $(1 \mathrm{~mm} \times 1 \mathrm{~mm} \times 1.8 \mu \mathrm{m})$ and two polysilicon supporting beams (length, width, and thickness being 400, 100 and $1.8 \mu \mathrm{m}$, respectively). Over the polysilicon plate lies an electroplated permalloy (Ni80 Fe20) plate $1 \mathrm{~mm} \times 1 \mathrm{~mm} \times 5 \mu \mathrm{m}$ in size. When an external magnetic field perpendicular to the assembly chip substrate is applied, the permalloy plates will align to the magnetic field lines, causing the flap to deflect out-of-plane (Fig. 2). 


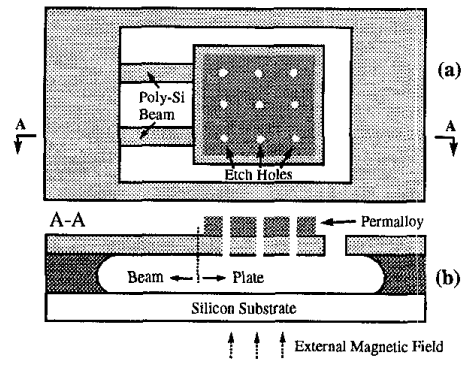

Fig. 1. Schematic of an out-of-plane permalloy magnetic actuator. (a) Top view; (b) cross-sectional view.
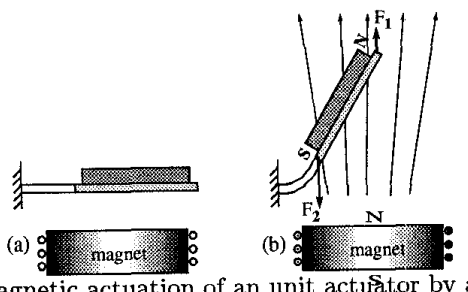

Fig. 2 Magnetic actuation of an unit actuator by an external electromagnet. (a) Rest position under zero external magnetic field; (b) out-of-plane actuation under a non-zero magnetic field. $F_{1}$ and $F_{2}$ are the modeling forces on the upper and lower edges of the plate.

Such an actuation can be used to lift and transport an object on top of the assembly chip. According to our proposed driving mechanism, there are two phases within a complete driving cycle (Fig. 3 ). In phase I, the flap is deflected at a slow speed and the free end of the flap moves both upward and side-ways, bringing the object with it. In phase II, as the magnetic field is removed, the flap and the object will both drop toward the substrate at different speeds. Assume that the field is removed quickly, the flap is subjected to both the gravitational and support-beam restoring forces. It drops to the substrate within $\sim 1.5 \mathrm{~ms}$, a time estimated by taking one quarter of the inverse of the the actuator's first-mode mechanical resonant frequency, obtained through finite-element modeling (ANSYS). The object, under the gravitational force alone, will drop to the substrate in $14 \mathrm{~ms}$ (assuming it is lifted by $1 \mathrm{~mm}$ ). It is therefore conceivable that the flaps and the object will separate in phase II, allowing the object to drop vertically in front of its original rest position. The object thus has a net horizontal displacement after each actuation cycle.

To use a group of actuators, flaps on a finished $1 \times 1$ $\mathrm{cm}^{2}$ die are arranged in four quadrants. Each quadrant contains a $7 \times 8$ array of actuators all aligned in a same direction (Fig. 4); directions of all four quadrants form a clockwise loop. Under current experimental requirements and conditions, all actuators in the four quadrants move at the same time under a uniform magnetic field (synchronous mode). The actuators are so arranged to verify a theory [1] that both translational (within each quadrant) and rotational motions (across two neighboring quadrants) of an object can be produced.

The actuators are fabricated by using a combined

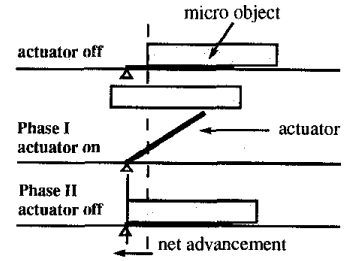

Fig. 3 Schematic diagram of synchronous driving of an object. (Top) Initial rest positions of an object and an unit actuator; (middle) elevated positions of the object and the actuator, after phase I; (bottom) positions at the end of a complete cycle. The object travels to the left (in phase I) and to the right (in phase II) by different amounts, therefore a net forward movement can be achieved.

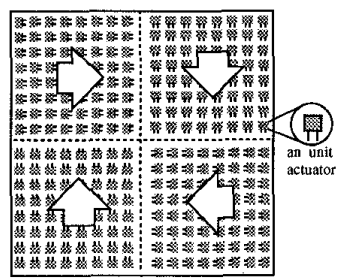

Fig. 4 The layout diagram of a $1 \times 1 \mathrm{~cm}^{2}$ actuator chip with micro-actuators arranged in four quadrants. The large arrows show the intended object-movement directions by actuators in show the intended object-movement directions by actuators in
each quadrant; directions in the four quadrants form a clockwise loop.

surface micromachining and permalloy electroplating technique [16], with major steps depicted in Fig. 5. SEM pictures of fabricated devices are shown in Figs. 6 and 7 .

\section{TESTING AND DISCUSSION}

Three types of experiments have been conducted: magnetic actuation of a unit actuator, lifting and dropping of objects, and in-plane translation/rotation of objects.

\section{Unit Actuator Characterization}

Inside a magnetic field, magnetic forces can be estimated by a simplified magnetic-charge model. Two point forces, $F_{1}$ and $F_{2}$, will develop on the two ends of the permalloy plate (Fig. 2), whereas $F_{1}=M_{s}(W T) H_{1}$ and $F_{2}=M_{s}(W T) H_{2} . \quad M_{s}$ is the permalloy satura-

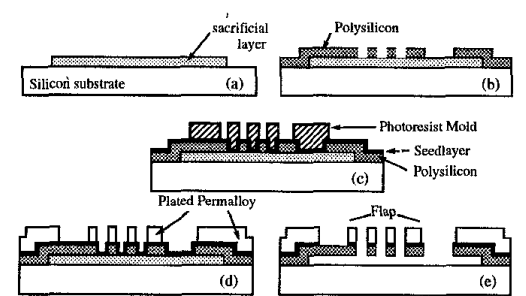

Fig. 5 Major fabrication steps of the micro-flaps. (a) A sacrificial material (phosphosilicate glass) is deposited and patterned over a silicon substrate; (b) polysilicon is deposited and plate/beam structures are patterned; (c) a $\mathrm{Cr} / \mathrm{Au}$ seed layer is evaporated and a thick photoresist electroplating mold is formed; (d) permalloy is electroplated and the photoresist/seedlayer removed; (e) the actuator is free-released by using concentrated $\mathrm{HF}$. 
tion magnetization, $H_{1}$ and $H_{2}$ are the magnetic field strengths near the top and bottom ends of the plate $\left(H_{2}>H_{1}\right)$, and $\mathrm{W}$ and $\mathrm{T}$ are the width and thickness of the permalloy plate. The vertical and angular displacements at the end of the flap are estimated using theoretical models $[14,15]$. To experimentally verify the theory, we studied the side profile of an individual actuator in a microscope monitoring system [12] where an electromagnet is used to provide the magnetic field. For this magnet, $H(\mathrm{~A} / \mathrm{m})=21.5 \times 10^{4}-3.13 \times 10^{4} \times d$ and $d$ (in $\mathrm{mm}$ ) is the distance from the measurement point to the surface of the magnetic core. Fig 8 . shows the measured angular and vertical deflections, together with theoretical calculations. The actuator can bend by more than $\sim 65^{\circ}$ and reach a maximum vertical deflection of $\sim 1.1 \mathrm{~mm}$ at its free end.

\section{Lifting and Dropping Weights}

We characterize the loading capacity of a unit actur ator by varying the magnetic field very slowly in both phases I and II. In this case, the object always follows the tip of the actuator and no horizontal movement is observed. Fig. 9 shows typical vertical deflections (normalized) vs. magnetic field $(H)$ curves for a same

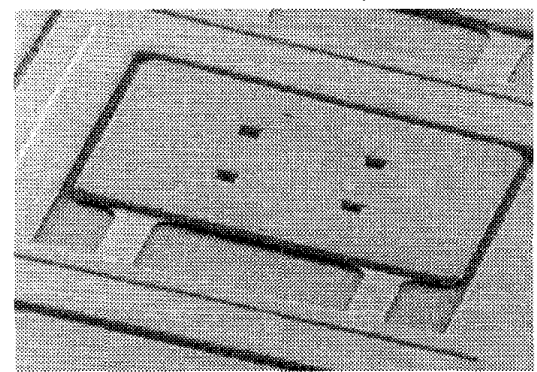

Fig. 6 An SEM micrograph of a fabricated magnetic flap. The size of the plate is $1 \times 1 \mathrm{~cm}^{2}$, the beams are $400 \mu \mathrm{m}$ long and $100 \mu \mathrm{m}$ wide.

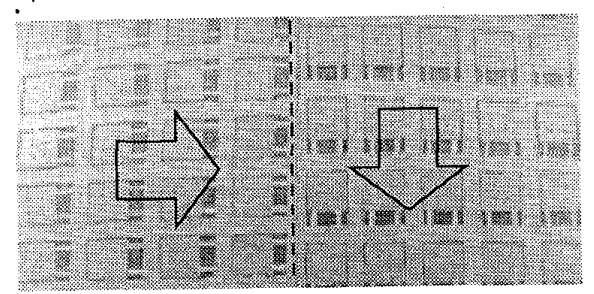

Fig. 7 A picture of two neighboring quadrants on a fabricated micro robotics assembly chip. Arrows indicate the ricated micro robotics assembly chip. Arrows indicate the the dashed line is the boundary of the two quadrants.
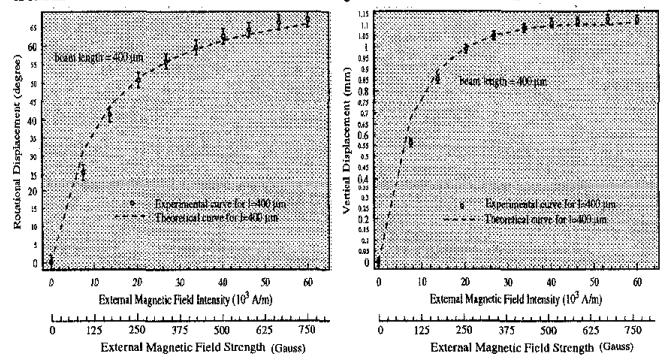

Fig. 8 Theoretical calculations and experimental data for rotation angle and maximum vertical deflection vs. applied magnetic field intensity H. A maximum $65^{\circ}$ of angular deflection and $1.1 \mathrm{~mm}$ of vertical displacement are achieved. group of actuators with and without a load. The load is a $20 \mathrm{mg}$ silicon chip sitting evenly over $2 \times 2$ flaps. Two phenomena are observed. First, deflection of a loaded flap occurs at a higher $H$ value compared with that of un-loaded flaps; secondly, a hysteresis between phase I and II movements is markedly exhibited for the loaded flaps. The first phenomenon occurs because the magnetic forces must exceed the gravitational force of the load to achieve its initiate flap deflection. The hysteresis between the curves of the two phases is believed to be due to the magnetization hysteresis of the permalloy material.

To measure the maximum weight-lifting capacity, we applied a magnetic field $\left(2 \times 10^{4} \mathrm{~A} / \mathrm{m}\right)$ to lift and drop a stack of silicon chips ( $222 \mathrm{mg}$, or $2.18 \mathrm{mN}$ ) using a $5 \times 5$ flap array (Fig. 10). This translate into roughly $87 \mu \mathrm{N}$ (or $8.88 \mathrm{mg}$ ) for each actuator, which has a mass of only $44.5 \mu \mathrm{g}$ itself. Such a loading capacity of the actuator arrays is very favorable for their intended applications.

\section{Elementary Object Positioning}

We also tested transportation of chips made of both glass and silicon. Using a surface profiler (Alphastep 200 ), the arithmetic average roughness is measured as $5 \mathrm{~nm}$ for the glass surface, $10 \mathrm{~nm}$ and $85 \mathrm{~nm}$ for the front and back side of the silicon chip, respectively. Fig. 11 shows the side view of a glass chip during one complete driving cycle. It it clearly shown that the chip gained a net horizontal movement, therefore

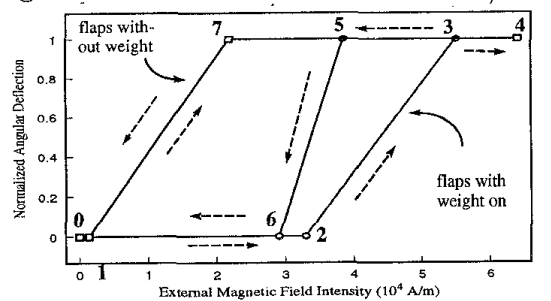

Fig. 9 Vertical deflection (normalized to the maximum displacement) vs. the magnetic field $H$. The trace $0-2-3-4$ is the phase-I curve and 4-5-6-0 is the phase II curve of a loaded actuator; traces $0-1-7-4$ and $4-7-1-0$ are the curves of a free flap during phases I and II, respectively.

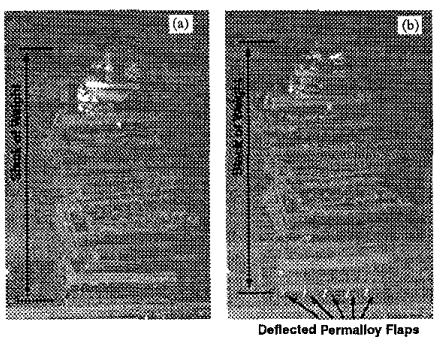

Fig. 10. A side-view of a stack of silicon chips (a) at their rest position when the actuators are off; (b) in a lifted position when the actuators are activated.

proving our intended operation mechanism. However, the actual mechanisms observed in our experiments are more complicated than what was proposed. Here, we discuss only two important phenomena.

The first phenomenon can be observed in Fig. 11. The edge of the glass chip at the lifted position (Fig. 11b) is different from that of the glass chip after phase II (Fig. 11c); the chip does not follow a straight vertical path when it is dropped. It is believed that during 
phase II, a horizontal momentum is imparted onto the glass chip by the frictional forces between the actuators and the chip; in other words, the actuators and the glass chip remain in contact during part of phase II. This point is again verified by experimentally measuring the finite decay time of the external magnetic field. The flap drops off to the substrate within $10 \mathrm{~ms}$, a time that is comparable with the $14 \mathrm{~ms}$ it takes for the glass chip to reach the substrate under the gravitational force alone. A second phenomenon occurs when the magnetic field is turned on very quickly during phase I. In some cases, the glass chip will gain excessive horizontal and vertical momentum, and can jump and slide over the actuator array in phase I. This type of motion, so called a jumping mode, would allow for more horizontal movement but is generally uncontrollable.

Finally, we experimented with a single silicon chip (with a mass of $4.6 \mathrm{mg}$ ) to demonstrate that a micro chip can be both translated and rotated using the actuator array in the jumping mode. Fig. 12 shows the positions of the micro-chip during a typical operation containing 8 lifting/dropping cycles; the chip is trans-

(a)

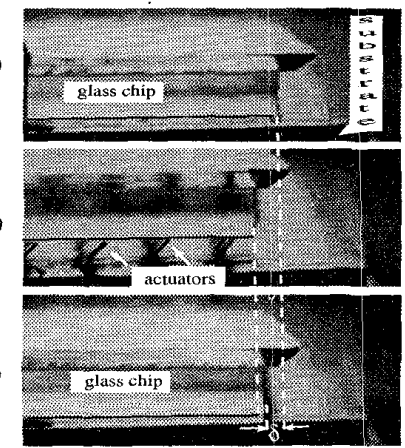

Fig. $11 \mathrm{~A}$ close-up view of an array of actuators during one synchronous driving cycle. (a) a glass chip $\left(3 \times 3 \times 0.9 \mathrm{~mm}^{3}\right)$ in its original rest position; (b) the position of the glass chip after phase I actuation, deflected actuators can be seen underneath the glass chip; (c) the position of the giass chip after phase II is completed. The difference in horizontal positions of the glass chip in (a) and (c), $\delta$, is the net movement achieved in one driving cvele.

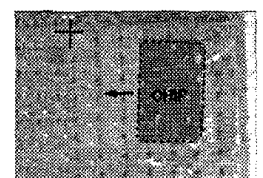

(a)

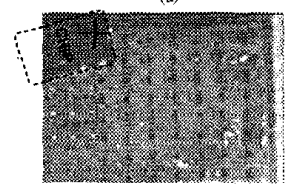

(c)

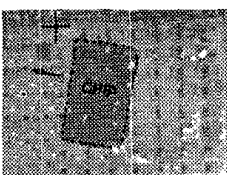

(b)

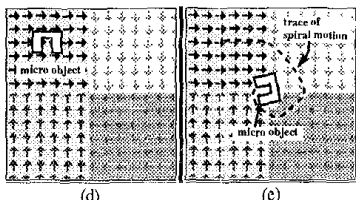

(d) lated by approximately $0.5 \mathrm{~mm}$ within the lower-right quadrant and rotated $70^{\circ}$ as it meets the boundary between two quadrants. The chip eventually moves toward the center of the four quadrants, completing a spiral trace as predicted by a computational analysis [1]. Note that longer translation and larger rotational angles have been demonstrated in other tests.

\section{CONCLUSION}

We have developed permalloy actuator arrays and experimentally verified the feasibility of a proposed synchronous driving mode. Lifting, holding, translating ard rotating chips made of glass and silicon have been demonstrated.

\section{REFERENCES}

1. P. Will, W.H. Liu, "Parts Manipulation on a MEMS Intelligent Motion Surface," paper submitted to the IEEE Transaction on Robotics and Automation.

2. K.F. Bohringer, B.R. Donald, R. Mihailovich, N. MacDonald "A Theory of Manipulation and Control for Micro Fabricated Actuator Arrays," Proceedings, IEEE Micro Electro Mechanical Systems, MEMS '94, pp. 102-107.

3. S. Konishi and H. Fujita, "System Design for Cooperative Control of Arrayed Microactuators," Proceedings, MEMS'95, pp. 322-327.

4. T. Akiyama and H, Fujita, "A Quantitative Analysis of Scratch Drive Actuator Using Buckling Motion," Proceedings, MEMS'95, pp. 310-315.

5. S. Konishi, H. Fujita, "A Conveyance System Using Air Flow Based on the Concept of Distributed Micro Motion Systems," J. of Microelectromechanical Systems, Vol. 3, No. 2, pp. 54-58, June 1994

6. K. S. J. Pister, R. S. Fearing, R.T. Howe, "A Planar Air Levitated Electrostatic Actuator System," Proceedings, MEMS '90, pp. $67-71$

7. M. Ataka, A. Omodake and H. Fujita, "A Biomimetic Micro Motion System," Proceedings, 7th International Conference on Solid-State Sensors and Actuators, Transducer '93, pp. 38-41. 8. I.J. Busch-Vishniac, "The Case for Magnetically Driven Microactuators," Sensors and Actuators, A 33, pp. 207-220, 1992.

9. C.H. Ahn and M.G. Allen, "A Fully Integrated Surface Micromachined Magnetic Microactuator with a Multilevel Meander Magnetic Core," IEEE J. Micro Electro Mechanical Systems, Vol. 2(1), pp. 15-22, 1993

10. H. Guckel, T.R. Christenson, K.J. Skrobis, T.S. Jung, J. Klein, K.V. Hartojo and I. Widjaja, "A First Functional Current Excited Planar Rotational Magnetic Micromotor," Proceedings, MEMS '93, pp. 7-11, 1993.

11. B. Wagner, W. Benecke, G. Engelmann and I. Simon, "Microactuators with Moving magnets for Linear, Torsional or Multiaxial Motion," Sensors and Actuators, A(32), pp. 598-603, 1992.

12. C. Liu, T. Tsao, Y.C. Tai and C.M. Ho, "Surface Micromachined magnetic Actuators," MEMS '94, pp. 57-62.

13. Y. Kim, M. Katsurai and H. Fujita, "A Levitation-type Linear Synchronous Microactuator using the Meisner Effect of High-Tc Superconductors," Sensors and Actuators, vol. A 29 , Nov. 1991, pp. $129-136$. 14. J.W. Judy, R.S. Muller and H.H. Zappe, "Magnetic Microactuation of Polysilicon Flexure Structures," 1994 IEEE Solid-State Sensor and Actuator Workshop, Hilton Head Island, SC, USA, pp. 43-48.

15. C. Liu, T. Tsao, Y.C. Tai, T.S. Leu, C.M. Ho, W. Tang and D. K. Miu, "Micromachined Permalloy Magnetic Actuator for Delta-Wing Control," MEMS'95, pp. 7-12.

16. V. Temesvary, S. Wu, W.H. Hsieh, Y.C. Tai and D.K. Miu, Design, Fabrication and Testing of Micromachined Electromagnetic Microactuators for Rigid Disk Drives," IEEE J. of Microelectromechanical Systems, Vol. 4, No. 1, pp. 18-27, March 1995. 